\title{
Short communication: Passive shedding of Mycobacterium avium ssp. paratuberculosis in commercial dairy goats in Brazil
}

\author{
D. G. G. Schwarz, ${ }^{*}$ M. C. Lima, ${ }^{*}$ M. Barros, ${ }^{*}$ F. L. Valente, ${ }^{*}$ T. M. Scatamburlo, ${ }^{*}$ N. Rosado, ${ }^{*}$ \\ C. T. S. A. M. Oliveira, $†$ L. L. Oliveira, $\ddagger$ and M. A. S. Moreira*1 \\ *Department of Veterinary, \\ †Department of Animal Science, and \\ łDepartment of General Biology, Universidade Federal de Viçosa (UFV), Av. P. H. Rolfs, s/n, CEP 36570-900, Viçosa, Minas Gerais, Brazil
}

\section{ABSTRACT}

Goat farming is a low-cost alternative to dairy production in developing countries. In Brazil, goat production has increased in recent years due in part to the implementation of programs encouraging this activity. Mycobacterium avium ssp. paratuberculosis (MAP) is the causative agent of paratuberculosis, a disease that causes chronic granulomatous enteritis in ruminants, but MAP transmission dynamics are still poorly understood in goats. In a previously published study of our research group, 10 dairy goat farms (467 animals) from Minas Gerais state were analyzed for MAP detection; 2 fecal cultures and 11 milk samples tested positive for MAP by conventional PCR and were confirmed by sequencing. Because no clinical signs were observed over $1 \mathrm{yr}$ of monitoring, we hypothesized that these MAPpositive goats could be passive shedders. Thus, in the present study, 4 positive goats (4/13) from the previous study were purchased and feces and milk samples were collected for evaluation (twice, with an interval of 3 mo between tests) by culture of MAP, IS 900 PCR, or both. All analyses were negative for MAP. At the last time point, blood samples were collected for ELISA, the animals were killed, and tissues collected for tissue culture and histopathology. At necropsy, no macroscopic lesions related to paratuberculosis were observed. Similarly, no histological changes were observed and MAP in samples stained by Ziehl-Neelsen was not detected. These animals were characterized as potential passive shedders with upward contamination of the teat canal by MAP. This is the first report of the passive shedding phenomenon in goats in Brazil and it highlights the importance of identifying these animals for control programs and to ensure the quality of dairy products.

Received March 23, 2017.

Accepted June 6, 2017.

${ }^{1}$ Corresponding author: masm@ufv.br
Key words: paratuberculosis, passive contamination, goat

\section{Short Communication}

During the last decade, goat production has increased globally (mainly in developing countries) due to its low cost and the fact that goat milk can serve as an alternative source of dairy food for people with an allergy to cow milk (Haenlein, 2004). Goat milk differs from cow milk by having smaller fat globules, less lactose, and more calcium, phosphorus, and potassium. Additionally, it has been suggested that goat milk has better digestibility because, at low $\mathrm{pH}$, it forms a finer curd than cow milk (Silanikove et al., 2010).

In 2013 , there were $207,008,068$ dairy goats worldwide and 6,799,000 in South America. This region is important because it is the highest goat milk producer in the American continent, at around 220,000 tonnes (FAO, 2016). Because of the increase in dairy goat farming, Brazil had become a leading producer of goat milk in the Americas, providing $69 \%$ of all goat milk produced in the continent (FAO, 2016). In this scenario, the increased production and the possibility of exporting live animals and milk products from goats have encouraged investigations into the control of diseases in these herds. The health of the dairy herd should be important to any producer, because without a healthy goat, there is no quality product, and there are no healthy goats without adequate hygienic control (Ribeiro and Ribeiro, 2010).

An important mycobacteriosis that affects ruminant herds around the world is paratuberculosis or Johne's disease, caused by Mycobacterium avium ssp. paratuberculosis (MAP). The infection results in a chronic granulomatous enteritis, diarrhea, progressive weight loss, malnutrition, a decrease in milk productivity, and death (Lombard, 2011). The progression of the infection results in MAP excretion in the feces, milk, or both. Therefore, feeding from infected dams facilitates 
the transmission of the infectious agent to young animals (Patel and Shah, 2011).

Most animals infected with MAP show a low and intermittent shedding pattern and only a few of them become high shedders (Mitchell et al., 2015). Frequently, the shedding patterns of natural MAP infection can be termed as passive shedding, where the animal swallows MAP organisms but is not infected (also referred to as the pass-through phenomenon), or active shedding, where MAP infects the intestinal epithelium and reaches the sub-epithelial macrophages (Kralik et al., 2014).

The present work is a continuation of a previous study by our research group where the presence of MAP was evaluated in 10 dairy goat farms from the mesoregion Zona da Mata, Minas Gerais state, Brazil. Among 467 animals (sampled from the previous study), 2 were positive by fecal culture and 11 were positive by conventional IS900 PCR performed on milk samples. These preliminary data were published by Souza et al. (2016). Over a period of $1 \mathrm{yr}$, we verified that these positive animals did not present any clinical signs suggestive of paratuberculosis. Considering this fact, we hypothesized that these animals could be passive shedders and their detection would be the first reported in Brazil.

In the present study, 4 positive goats ( 1 with positive fecal and milk samples and 3 with positive milk samples) from goat farms evaluated by Souza et al. (2016) and 4 controls (no MAP detection) from goat farms without the disease were purchased and maintained in separate stalls at the Veterinary Department, Universidade Federal de Viçosa (UFV), Brazil. All animal procedures followed the rules of the ethics committee on animal use (CEUA/UFV, protocol number 26/2014). Fecal and milk samples were collected twice, with an interval of 3 mo between them. At the second time point, blood samples were collected and, after the animals had been culled, fragments of ileocecal valve, colon, ileum, and mesenteric lymph nodes were collected for tissue culture and histopathology.

The processing of the fecal samples followed the method described by Stabel (1997) and Souza et al. (2016). Briefly, $2 \mathrm{~g}$ of feces was placed in a $50-\mathrm{mL}$ tube containing $20 \mathrm{~mL}$ of autoclaved distilled water and shaken at $110 \mathrm{rpm}$ for $60 \mathrm{~min}$ and then kept at rest for $45 \mathrm{~min}$ at room temperature for sedimentation. For decontamination, $5 \mathrm{~mL}$ of the supernatant was moved to a $50-\mathrm{mL}$ tube containing $20 \mathrm{~mL}$ of $0.9 \%$ hexadecylpyridinium chloride (HPC; Sigma-Aldrich, St. Louis, MO) and left at room temperature overnight. Then, the tubes were centrifuged at $1,700 \times g$ for $20 \mathrm{~min}$, the supernatant was discarded, and the pellet was resuspended in $1 \mathrm{~mL}$ of antimicrobial solution containing nalidixic acid $(50 \mathrm{mg} / \mathrm{L})$, vancomycin hydrochloride $(50 \mathrm{mg} / \mathrm{L})$, and amphotericin B $(150 \mathrm{mg} / \mathrm{L})$. A $150-\mu \mathrm{L}$ aliquot was inoculated onto 2 slanted tubes of Middlebrook (MB) $7 \mathrm{H} 11$ with mycobactin $\mathrm{J}(2 \mathrm{mg} / \mathrm{L})$ and 2 without the siderophore. The tubes were then incubated at $37^{\circ} \mathrm{C}$ and examined for MAP growth for up to 12 mo.

Milk samples were collected following strict hygiene standards. Before sampling, the udder was thoroughly washed and dried with a paper towel, and the teats were sprayed with $70 \%$ ethanol. The first 3 milk jets were discarded and the next $15 \mathrm{~mL}$ was collected in a sterile container. For MAP culture, the milk was processed according to Pillai and Jayarao (2002). Briefly, milk samples were centrifuged at $2,000 \times g$ for 15 min at $4^{\circ} \mathrm{C}$, the supernatant was discarded, and the pellet was resuspended in $15 \mathrm{~mL}$ of $0.75 \% \mathrm{HPC}$ at room temperature for $5 \mathrm{~h}$. Subsequently, samples were centrifuged at 2,000 $\times g$ for $15 \mathrm{~min}$ and the supernatant was discarded. Finally, the remaining pellet was resuspended in $600 \mu \mathrm{L}$ of antimicrobial solution and a $150-\mu \mathrm{L}$ aliquot was inoculated onto MB7H11 slopes with or without mycobactin $\mathrm{J}(2 \mathrm{mg} / \mathrm{L})$ and incubated in duplicate at $37^{\circ} \mathrm{C}$ for up to $12 \mathrm{mo}$.

For the molecular analysis, the milk samples were centrifuged at $2,000 \times g$ for $15 \mathrm{~min}$ at $4^{\circ} \mathrm{C}$ and, after discarding the supernatant, the pellet was resuspended in $200 \mu \mathrm{L}$ of $1 \%$ PBS, pH 7.2, and stored for molecular analysis. Extraction of genomic DNA from milk samples was performed using the Wizard Genomic DNA purification Kit (Promega, Madison, WI), according to the manufacturer's instructions. The DNA was resuspended in $30 \mu \mathrm{L}$ of DNA rehydration solution and its concentration was quantified by a NanoDrop Lite spectrophotometer (Thermo Fisher Scientific, Waltham, MA). Conventional IS 900 PCR reactions were performed using the primers BN1 (5'-GTTATTAACGACGACGCGGAGC-3') and BN2 (5'-ACGATGCTGTGTTGGGCGTTAG-3') (Sivakumar et al., 2005) and GAPDH forward (5'-GGCGTGAACCACGAGAAGTA- $3^{\prime}$ ) and reverse (5'-GGCGTGGACAGTGGTCATAA- $3^{\prime}$ ) as the internal control target for PCR reaction. Briefly, 12.5 $\mu \mathrm{L}$ of Go Taq Green Master Mix $2 \times$ (Promega), $1 \mu \mathrm{L}$ of each oligonucleotide, $6.5 \mu \mathrm{L}$ of MilliQ water, and $4 \mu \mathrm{L}$ of DNA at a concentration of $200 \mathrm{ng} / \mu \mathrm{L}$ were used in a final volume of $25 \mu \mathrm{L}$. The PCR was performed with initial denaturation at $94^{\circ} \mathrm{C}$ for $4 \mathrm{~min}, 30$ cycles of $94^{\circ} \mathrm{C}$ for $1 \mathrm{~min}, 60^{\circ} \mathrm{C}$ for $1 \mathrm{~min}$, and $72^{\circ} \mathrm{C}$ for $1 \mathrm{~min}$ and final extension at $72^{\circ} \mathrm{C}$ for $4 \mathrm{~min}$. The amplified fragments were visualized by $1 \%$ agarose gel electrophoresis (Invitrogen, Washington, DC) in Tris-borate-EDTA buffer stained with GelRed Nucleic Acid Gel Stain (Biotium, Hayward, CA) using an EagleEye II transilluminator (Stratagene, La Jolla, CA). The MAP K-10 strain was 
used as a positive control and ultra-pure water as the negative control. A 100-bp DNA ladder (Promega) was used as a molecular weight standard.

To evaluate the serology of the goats, serum from each animal was sent to Universidade Federal de Campina Grande, Brazil, to perform ELISA tests with the Idexx MAP Ab Test Kit (Idexx, Westbrook, ME) following the manufacturer's instructions.

For tissue culture, the methodology described by Greig et al. (1999) was followed, with modifications. Approximately $4 \mathrm{~g}$ of tissue was macerated in a mortar and pestle and homogenized with $2 \mathrm{~mL}$ of $0.9 \%$ saline. Then, the solution was transferred to $50-\mathrm{mL}$ tubes, treated with $0.9 \% \mathrm{HPC}$, and inoculated in MB7H11 slope tubes with and without mycobactin $\mathrm{J}$ as described above.

Histopathological analyses were performed using an established methodology whereby tissues were fixed in $10 \%$ formalin buffer solution for $24 \mathrm{~h}$ for further processing to obtain paraffin wax blocks. All sections were cut at $4 \mu \mathrm{m}$ and stained with hematoxylin and eosin for lesion analysis and with Ziehl-Neelsen (acid-fast) stain for MAP detection.

No characteristic colonies of MAP on MB7H11 were observed in any of the samples (feces, milk, and tissue) and no amplified fragments of a similar size (626 bp) to that expected from the MAP gene were observed in conventional PCR on milk samples. An internal control was used to ensure there was no inhibition of the PCR reaction, and no such inhibition of the $\mathrm{PCR}$ reactions was detected. The immune assay did not show any positive reaction to MAP in any of the analyzed serum samples. Macroscopic analyses of the organs did not show any characteristic alterations typical of paratuberculosis. Similarly, no histological changes were observed in the analyzed organs, and MAP was not detected in samples stained with Ziehl-Neelsen.

Few studies have identified passive shedders because of the difficulty in identifying this phenomenon in dairy herds. We know that the animals previously identified as positive by Souza et al. (2016) released MAP through milk and feces, because all analyzed samples in the earlier study were confirmed by sequencing to have 94 to $99 \%$ similarity to the MAP K-10 strain. However, in the current study, even using different standard techniques for MAP identification, analyses did not identify this microorganism in the samples. Recently, Mitchell et al. (2015) found that most naturally infected cows had a pattern of low and intermittent shedding that rarely progressed to high shedding. Although it is known that the preclinical phase of paratuberculosis in goats may be long and MAP shedding intervals can be highly irregular, we used a 1-yr period after detection of MAP and an additional 3 mo for evaluation. To avoid the premature culling of false-positive animals, it is recommended that consecutive exams be carried out to detect animals that are passive shedders. AlMajali et al. (2008) verified that the inclusion of new animals in a herd and the joint raising of goats and sheep increase the risk of paratuberculosis in the herd. Moloney and Whittington (2008) observed that when 1,774 cattle were raised with a MAP-positive sheep flock, only one cow became positive for the sheep strain (S) and was identified as a passive shedder. Similarly, Souza et al. (2016) demonstrated by restriction enzyme analysis that the positive goats in our study were identified as bearing the cattle strain $(\mathrm{C})$. There is a lack of information in the literature regarding the relationship between the host and the type of strains ingested. However, ingestion of non-species-specific MAP strains could intensify the occurrence of passive shedders.

Considering the properties evaluated in the present study, a previous investigation conducted by our research group identified MAP (unpublished data) in $50 \%$ of water samples for goat consumption and in $30 \%$ of water samples for human consumption. These data are associated with intensive goat production, which increases the risk of ingestion of contaminated feed and the occurrence of the passive shedding phenomenon. In addition, these animals may be a source of contamination for humans, because MAP has been detected in dairy products such as pasteurized cow milk and retail artisanal Coalho cheese for human consumption (Carvalho et al., 2012b; Faria et al., 2014). Although the zoonotic potential of MAP is not yet clear, it has a historical association with Crohn's disease due to similarities between the lesions and clinical signs in animals and humans, as well as the detection of the microorganism in patients with Crohn's disease (Abubakar et al., 2008; Carvalho et al., 2012a; Timms et al., 2016).

These findings are important for dairy production because they demonstrate, for the first time in Brazil, that goats can spread MAP in the environment via their feces and infect susceptible animals passively through milk. However, it should be considered that these goats may have been infected but have cleared the infection completely or that they remained infected but the infection was contained by the immune response. This investigation reconfirms the difficulty in identifying MAP-infected goats during the preclinical phase of paratuberculosis and reveals how urgently new diagnostic approaches are needed. Furthermore, prior MAP detection in milk (using PCR) and subsequent negativity suggests that MAP could enter the teat canal from the environment, contaminating the milk but not infecting the animal.

The present study describes, for the first time in Brazil, goats that could be passive shedders and provides 
evidence of upward contamination of the teat canal by MAP in dairy goats from Zona da Mata (Minas Gerais, Brazil). These findings highlight the importance of identifying these animals for control programs and to ensure the quality of dairy products.

\section{ACKNOWLEDGMENTS}

The authors acknowledge the Sergio Santos de Azevedo for performing the ELISA tests in his laboratory in the Universidade de Campina Grande/PB and the financial support from $\mathrm{CNPq}$ (Conselho Nacional de Desenvolvimento Científico e Tecnológico, Brasília, Brazil), FAPEMIG (Fundação de Amparo à Pesquisa de Minas Gerais, Belo Horizonte, Brazil), and CAPES (Coordenação de Aperfeiçoamento de Pessoal de Nível Superior, Brasília, Brazil). M. A. S. Moreira is supported by CNPq.

\section{REFERENCES}

Abubakar, I., D. Myhill, S. H. Aliyu, and P. R. Hunter. 2008. Detection of Mycobacterium avium subspecies paratuberculosis from patients with Crohn's disease using nucleic acid-based techniques: A systematic review and meta-analysis. Inflamm. Bowel Dis. 14:401-410.

Al-Majali, A., K. Jawasreh, and A. A. Nsour. 2008. Epidemiological studies on foot and mouth disease and paratuberculosis in small ruminants in Tafelah and Ma'an, Jordan. Small Rumin. Res. 78:197-201.

Carvalho, I. A., M. L. Ferrari, and M. A S. Moreira. 2012a. Mycobacterium avium ssp. paratuberculosis vs Crohn's disease. Pages 129-142 in Crohn's Disease. Vol. 1. S. Karoui, ed. Intech, Rijeka, Croatia.

Carvalho, I. A., P. A. Pietralonga, D. G. G. Schwarz, A. C. S. Faria, and M. A. S. Moreira. 2012b. Short communication: Recovery of viable Mycobacterium avium subspecies paratuberculosis from retail pasteurized whole milk in Brazil. J. Dairy Sci. 95:6946-6948.

FAO. 2016. FAOSTAT: Livestock Primary. Food and Agriculture Organization of the United Nations. Accessed Nov. 3, 2016. http:// faostat.fao.org/beta/en/\#data/QL

Faria, A. C., D. G. Schwarz, I. A. Carvalho, B. B. Rocha, K. N. C. Castro, M. R. Silva, and M. A. Moreira. 2014. Short communication: Viable Mycobacterium avium subspecies paratuberculosis in retail artisanal Coalho cheese from northeastern Brazil. J. Dairy Sci. 97:4111-4114.

Greig, A., K. Stevenson, D. Henderson, V. Perez, V. Hughes, I. Pavlik, M. E. Hines, I. Mckendrick, and J. M. Sharp. 1999. Epidemiological study of paratuberculosis in wild rabbits in Scotland. J. Clin. Microbiol. 37:1746-1751.

Haenlein, G. F. W. 2004. Goat milk in human nutrition. Small Rumin. Res. 51:155-163.

Kralik, P., R. Pribylova-Dziedzinska, A. Kralova, K. Kovarcik, and I. Slana. 2014. Evidence of passive faecal shedding of Mycobacterium avium ssp. paratuberculosis in a Limousin cattle herd. Vet. J. 201:91-94.

Lombard, J. E. 2011. Epidemiology and economics of paratuberculosis. Vet. Clin. North Am. Food Anim. Pract. 27:525-535.

Mitchell, R. M., Y. Schukken, A. Koets, M. Weber, D. Bakker, J. Stabel, R. H. Whitlock, and Y. Louzoun. 2015. Differences in intermittent and continuous fecal shedding patterns between natural and experimental Mycobacterium avium subspecies paratuberculosis infections in cattle. Vet. Res. 46:66. https://doi.org/10.1186/ s13567-015-0188-x.

Moloney, B. J., and R. J. Whittington. 2008. Cross species transmission of ovine Johne's disease from sheep to cattle: An estimate of prevalence in exposed susceptible cattle. Aust. Vet. J. 86:117-123.

Patel, A., and N. Shah. 2011. Mycobacterium avium subsp paratuberculosis-incidences in milk and milk products, their isolation, enumeration, characterization, and role in human health. J. Microbiol. Immunol. Infect. 44:473-479.

Pillai, S. R., and B. M. Jayarao. 2002. Application of IS900 PCR for detection of Mycobacterium avium subsp. paratuberculosis directly from raw milk. J. Dairy Sci. 85:1052-1057.

Ribeiro, A. C., and S. D. A. Ribeiro. 2010. Specialty products made from goat milk. Small Rumin. Res. 89:225-233.

Silanikove, N., G. Leitner, U. Merin, and C. G. Prosser. 2010. Recent advances in exploiting goat's milk: Quality, safety and production aspects. Small Rumin. Res. 89:110-124.

Sivakumar, P., B. N. Tribathi, and N. Singh. 2005. Detection of $M y-$ cobacterium avium subsp. paratuberculosis in intestinal and lymph node tissues of water buffaloes (Bubalus bubalis) by PCR and bacterial culture. Vet. Microbiol. 108:263-270.

Souza, M. C. C., M. C. Lima, I. F. E. Braga, D. G. G. Schwarz, A. N. S. Rodrigues, E. B. Sales, A. N. F. Junior, and M. A. S. Moreira. 2016. Molecular typing of Mycobacterium avium ssp. paratuberculosis (MAP) isolated from dairy goats in Brazil. Small Rumin. Res. 140:18-21.

Stabel, J. R. 1997. An improved method for cultivation of Mycobacterium paratuberculosis from bovine fecal samples and comparison to three other methods. J. Vet. Diagn. Invest. 9:375-380.

Timms, V. J., G. D. Hazel, M. Mitchell, and B. A. Neilan. 2016. The association of Mycobacterium avium ssp. paratuberculosis with inflammatory bowel disease. PLoS One 11:e0148731. 